\title{
Development of Cloud Computing Platform Based on Neural Network
}

\author{
Zhi Zhang, Liwei Wang $\mathbb{D}^{\mathbb{D}}$, Ruiying Liu, and Jinghang Fan \\ State Grid Hebei Information \& Telecommunication Branch, Shijiazhuang, Hebei 050000, China \\ Correspondence should be addressed to Liwei Wang; 15030720552@xs.hnit.edu.cn
}

Received 10 January 2022; Revised 9 February 2022; Accepted 14 February 2022; Published 7 March 2022

Academic Editor: Naeem Jan

Copyright (c) 2022 Zhi Zhang et al. This is an open access article distributed under the Creative Commons Attribution License, which permits unrestricted use, distribution, and reproduction in any medium, provided the original work is properly cited.

\begin{abstract}
Aiming at the problems of small data storage, small platform throughput, and high energy consumption in the development of existing cloud computing platforms, this paper develops a cloud computing platform based on neural networks. In the design of the platform, firstly, the functions of the cloud computing platform are determined. Based on the function design, the hardware and software of the cloud computing platform are designed. In the hardware design, the topology of cloud computing platform, data acquisition module, single-chip microcomputer, node deployment, and other types of functional hardware are designed. In the software design, the neural network is mainly used to remove the redundancy of data stored in a cloud computing platform, design the data processing flow of cloud computing platform, and complete the development of cloud computing platform based on neural network. The experimental results show that the cloud computing platform based on the neural network designed in this paper runs faster, the throughput of platform data has been significantly improved, and the operating energy consumption of the platform is low.
\end{abstract}

\section{Introduction}

With the development of information technology, the Internet has become an indispensable part of people's life. People cannot get information, publish information, and exchange information without the Internet. In this context, the computing that consumes the most CPU is gradually transferred to the processing of information [1]. The computing power that a single processor can provide is close to the limit. This makes major manufacturers face great challenges; they need to mine useful information from TB and even $\mathrm{Pb}$ level data and process this information quickly and efficiently [2].

Cloud computing technology is a business model rising in recent years [3]. It combines the characteristics of distributed computing, parallel computing, and grid computing and uses the computing cluster built by a large number of ordinary servers and the storage cluster built by a large number of low-cost devices to provide users with scalable computing resources and storage space. Cloud computing technology creates a new application mode, which makes the computing power circulate through the network and provide computing power services comparable to supercomputers through combination, and gradually develops into a network application trend. The deployment and application of supercomputer applications face a very high threshold due to the very expensive hardware investment, while cloud computing combines ordinary personal computers and standard servers into computer clusters through the Internet, becoming a low-cost solution for supercomputing services [4]. In the cloud computing environment, both the number of users and the number of resources are very large. Without a rigorous regulatory mechanism, it is easy to be chaotic and out of order. In addition, China's laws and regulations on intellectual property are not perfect, piracy is very serious, and the intellectual property rights of resource builders are vulnerable to infringement. In order to ensure the smooth progress of resource sharing in the cloud environment, it is necessary to establish a rigorous regulatory mechanism to supervise the sharing behavior of users. Establishing a professional organization with organizational ability and supervision is a better solution [5]. 
The neural network has been the research hotspot of artificial intelligence since the 1980s. Its accuracy in many applications of artificial intelligence is higher than that of traditional methods. A neural network is composed of a large number of interconnected nodes, and the connection also contains many nonlinear elements. A large number of nodes need a large number of parameters. Neural network training is the process of constantly adjusting network parameters. Neural network training often needs many iterations to optimize the network performance, which usually takes a lot of time. Even using a graphics processor to accelerate neural network training is still very time-consuming. In addition, neural network training often needs a lot of training data. A neural network needs a lot of training data and time-consuming computing, which makes it very suitable for cloud computing platforms [6]. The cloud computing platform can store a large number of training data in a distributed manner and can also use multiple cloud nodes to participate in the calculation of neural networks at the same time. Compared with the neural network running on a single machine, the neural network based on a cloud computing platform greatly shortens the training time and has the ability to store and process massive training data. Aiming at the large amount of data stored in the network, this paper develops a cloud computing platform based on a neural network to improve the effectiveness of network data storage.

The arrangements of this paper are as follows: Section 1 consists of an introduction. Section 2 discusses the development of a cloud computing platform based on a neural network. Section 3 introduced the experimental analysis as well as an experimental scheme, design of experimental indicators, and analysis of experimental results in detail. Section 4 concludes the paper.

\section{Development of Cloud Computing Platform Based on Neural Network}

The delivery of various services over the Internet is known as cloud computing. These resources include data storage, servers, databases, networking, and software, among other tools and applications. As long as an electronic device has Internet access, it has access to the data as well as the software programs needed to run it.

\subsection{Function Analysis of Cloud Computing Platform}

2.1.1. Application and Management of the Virtual Machine. The application includes the selection of virtual machine type and image, as well as the naming of the virtual machine. Management mainly includes virtual machine deletion, virtual machine suspension/recovery, snapshot creation, and virtual machine monitoring [7].

2.1.2. Snapshot Management. Snapshots created by users are displayed here in the form of a list, which can be deleted, or a new virtual machine can be started directly from the snapshot. In addition, the administrator can also see and manage the backup snapshots of each virtual machine of the user.

2.1.3. Full Rule Management. Users can create their own security rules and assign different security rules to different virtual machines rules to provide a more flexible firewall.

2.1.4. Management of Physical Hosts. View the running status of the physical. And you can wake up the physical host Make. Check the number of virtual machines running in the host, and perform hot migration for physical machines with low resource occupancy to save energy.

2.1.5. Load Forecasting. According to the load of the virtual machine in the past month, predict the load of the virtual machine in the next 5 days. According to the situation, users can allocate or recycle virtual machines in time.

2.1.6. Load Monitoring. Users can view a load of their virtual machines in the past day, and administrators can also view objects that manage the load of the host in the past day.

Different service resource types are provided by cloud computing; the industry will be divided into three service categories: platform as a service (PaaS), software as a service (SaaS), and infrastructure as a service (IaaS) [8]. PaaS allows users to deploy applications created by users on the infrastructure or obtain applications created using programming languages, services, and tools supported by the provider; SaaS allows users to use the application running on the cloud infrastructure by the provider; IaaS provides users with the ability to equip processing, storage, network, and its basic computing resources where the software is deployed and run, including the operating system and application programs. Open Stack is an open-source IaaS implementation. It is a key Cloud Computing Development Project cosponsored by NASA Rackspace. It integrates a series of interrelated open-source groups to form a seamless selfservice platform and provides high-performance computing, store, and network by using virtualization and scalable technology [9].

\subsection{Hardware Design of Cloud Computing Platform}

2.2.1. Topology Design of Cloud Platform. The topology design of a cloud platform is related to the overall development of the platform. Cloud platform users access the web-based cloud platform management system through the enterprise internal network. Users are divided into administrators and ordinary users according to their permissions. Administrator users manage cloud platform resources through the cloud platform management system, and ordinary users can apply for resources through the cloud platform management system [10]. The cloud platform management system calls the resource scheduling interface to operate virtual machines, physical hosts, and other resources. It encapsulates all the interfaces for operating Open Stack and the shutdown/wake-up interface of physical hosts 
[11]. The prediction server obtains and monitors the running load data of physical hosts and virtual machines and stores the data in the Mongo database. In addition, the MongoDB database also stores the relevant information of users in the cloud platform management system [12]. The topology of the cloud platform is shown in Figure 1.

2.2.2. Design of Data Acquisition Module. In the hardware design of the cloud computing platform, the platform is developed based on the existing hardware. Its main function is to effectively collect and store data [13]. Therefore, this paper first designs the automatic data acquisition module for massive network resources. The design flow of the acquisition module is shown in Figure 2.

Through wireless transmission, the data in different environments in the network can be monitored in real time, the abnormal state of data can be paid attention to in real time, and early warning can be carried out. In this process, through the effective realization of monitoring. When the data in the network is monitored in real time, the collected characteristic data is transmitted to the main control room [14].

2.2.3. Single-Chip Microcomputer Selection of Cloud Computing Platform. In the hardware design of cloud computing platforms, in order to ensure the performance of cloud computing platforms, the selection of a singlechip microcomputer is very important. As the platform terminal control core, stm32f103zet6 is the hardware support of this paper. The single-chip microcomputer is composed of a 64-bit enhanced microprocessor, including 244 pins. Its main frequency is $128 \mathrm{MHz}$. Its powerful function can be used as the application of the design platform in this paper [15]. In order to ensure the accessibility and expansibility of the system, the multifunctional embedded performance of the single-chip microcomputer is good, including multiple $\mathrm{I} / \mathrm{O}$ ports. The access port mode is shown in Figure 3.

Each interface set can be effectively connected with the software to realize a convenient connection. The MCU also has rich peripheral resources, with multiple high-speed ADC and RTC real-time clocks. The clock is the hardware basis of the terminal, which can effectively improve the integration of the system, reduce the use of special chips for peripherals, and reduce the system cost.

\subsubsection{Design of Core Controller of Cloud Computing} Platform. With the passage of time and the increasing data of cloud computing platforms, it is necessary to set the master controller to improve the performance of the platform control capability to meet the control requirements of the platform. This paper takes the tc1782 microcontroller as the core controller [16]. The controller includes 14 input channels, 4 communication structures, and arbitrarily configurable interface circuits. The interface circuit is shown in Figure 4.
2.2.5. Node Deployment Design in Cloud Computing Platform. Node deployment in a cloud computing platform is related to the function realization of the whole platform design. Open Stack is built by its subsystems. Each subsystem can be installed separately, so there are various ways to build it, but generally speaking, it is divided into two types: one is all in one, and the other is multinodes. Single node installation installs all subsystems on a physical host, which is generally used for introductory learning or testing, not really into the production environment. Multinode installation is to install different subsystems on different physical hosts according to needs to provide flexible functional services. In this cloud platform, Open Stack mainly uses three functions: image management, security group management, and virtual machine management. Three core subsystems of Open Stack are deployed on the node, namely, the authentication subsystem keystone, the image subsystem glance, and the Nova management-related components of the virtual machine subsystem Nova [17]. In addition, MySQL database and quid message queue are installed as service support. On the computing node, deploy the Nova network component, and Nova computes the component of the subsystem Nova [18]. The specific deployment method is shown in Figure 5.

\subsection{Software Design of Cloud Computing Platform Based on} Neural Network. A neural network simulates the human brain neural network to establish a model, which can be logically understood as the approximation of a certain algorithm or function. A neural network is widely used in the field of artificial intelligence and shows good results. In order to make the neural network model fit the target task as much as possible, the model parameters need to be adjusted continuously during training. Each training of neural network is to analyze the error between the output of the network and the expected output and appropriately adjust the network parameters according to the error [19] so that the model can better fit the data. The gradient descent method is the most commonly used optimization algorithm for depth neural networks. The error between the output of the neural network and the expected output is defined as a loss function. The gradient descent method calculates the derivative of the loss function to the parameter and adjusts the parameter along the opposite direction of the derivative.

Assuming that the loss function is a derivative of the parameter $\stackrel{\Delta}{\longrightarrow} A$, the new weight result is

$$
\stackrel{\Delta}{\longrightarrow} A=A-R \times \nabla H .
$$

Among them, $R$ represents the learning rate, the value affects the distance that the parameter moves in the opposite direction of the derivative, and the loss function of the modified neural network will reach the optimal value.

The neural network has a large number of parameters, and the direct calculation of the partial derivative of the loss function has a huge amount of calculation and low efficiency. The propagation link of the backpropagation 


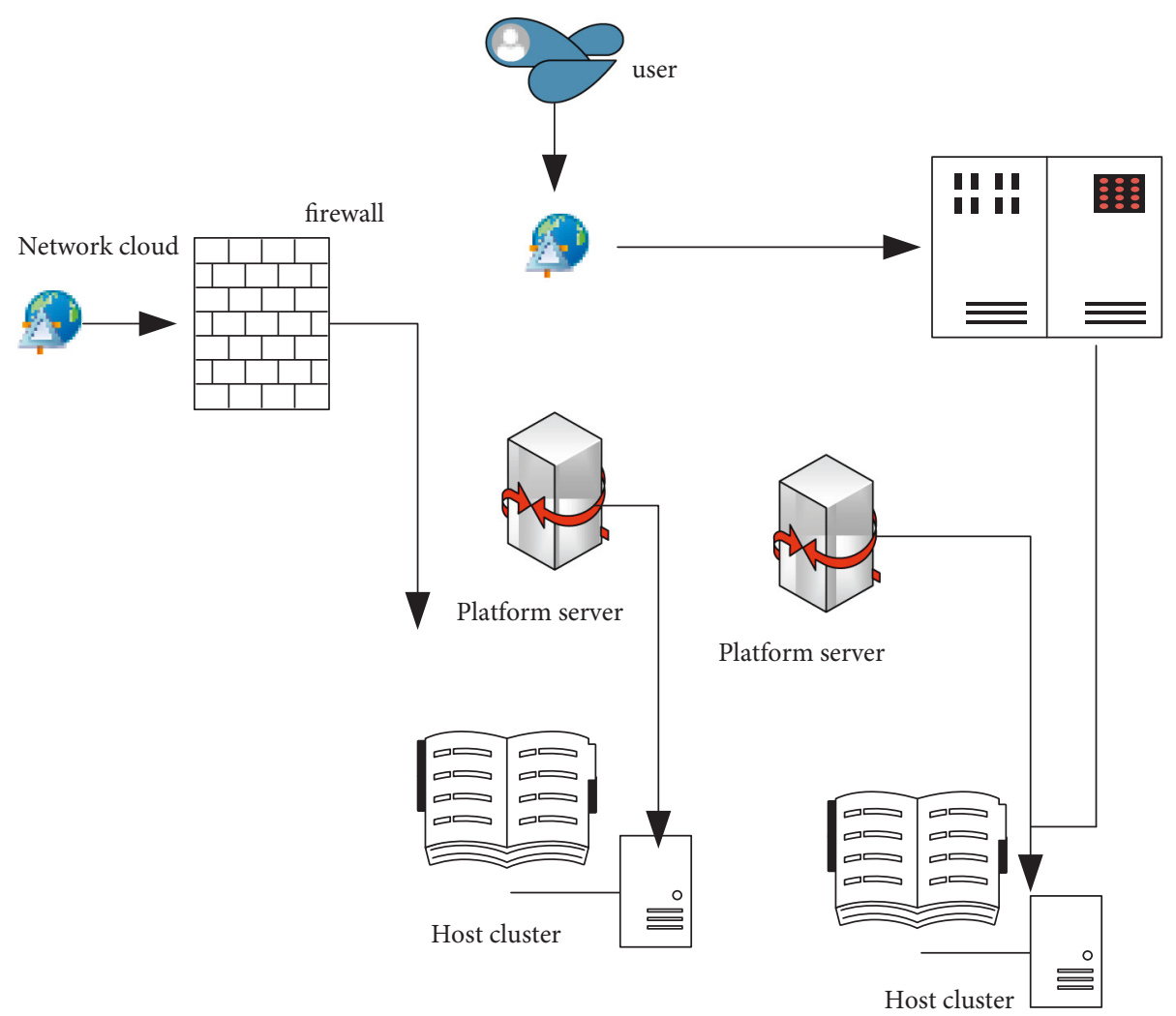

Figure 1: Topology of cloud platform.

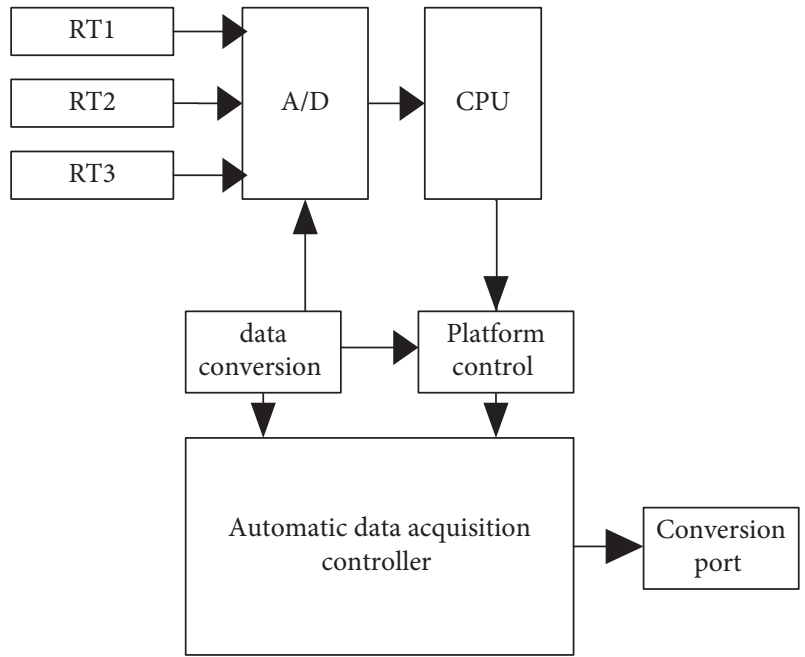

FIgURe 2: Acquisition module design.

algorithm includes two stages: the forward propagation stage and the backpropagation stage. In the forward propagation stage, the input is passed through the neural network, and the output is obtained. The backpropagation stage follows the chain rule, which takes the output of the forward propagation as the input and the input as the output, and passes the derivation process back to each layer of the neural network in a progressive way. The gradient descent algorithm in a neural network adopts backpropagation to improve the efficiency of calculation.
In this paper, in the design of a cloud platform, the neural network is applied to effectively process the resource data in the platform, reduce the work complexity of the platform, and improve its reflection speed [20].

In the cloud platform resource storage, resources are mainly stored to support the operation of the cloud platform. Due to the huge number of resources and many duplicate resource data, the resource data stored in the module needs to be processed. The resource data is fused to reduce the duplication of resource data. The resource data is clustered by the hierarchical clustering algorithm, the same attribute data is set as a cluster, then the similarity of any two resource data in the cluster family is compared, the two data with the highest similarity are combined, and the process is repeated until the similarity in the data is the lowest. The specific steps are as follows.

Assuming that the cluster category of the resource data is set to $E_{i}, E_{j}$, the similarity of both data was determined based on the characteristics of the resource data in these two categories, and $t$ is

$$
H\left(E_{i}, E_{j}\right)=R S_{E_{i}}\left(E_{i}, E_{j}\right)+\rho S_{E}\left(E_{i}, E_{j}\right) .
$$

The formula represents the degree of resource data in the two clusters, $\rho$ is the degree of similar weight of each resource data, and $H_{E_{i}}\left(E_{i}, E_{j}\right)$ is the difference of resource data.

Suppose that there are two categories in the class, analyze the similarity between them, and express them according to the characteristics of data attributes and structural relationships within the data. 


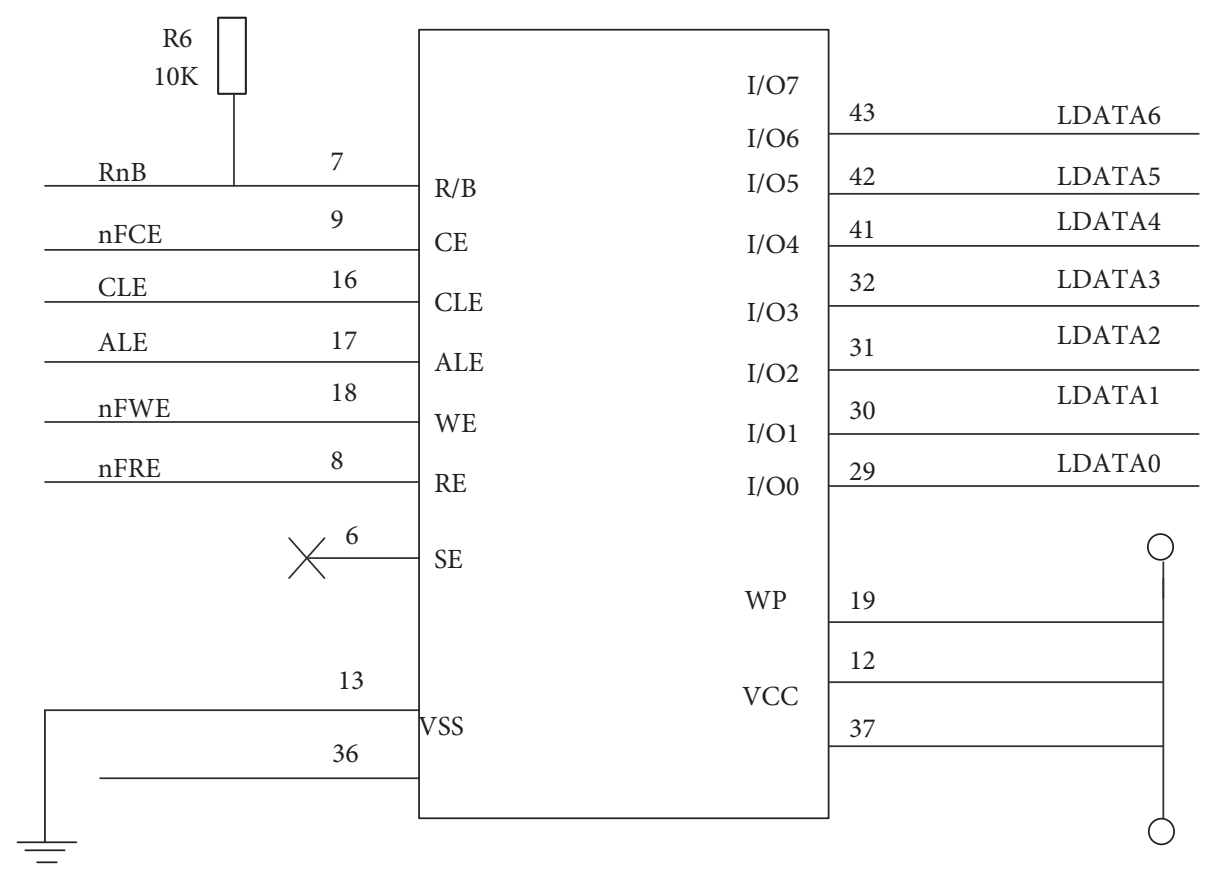

Figure 3: Single-chip microcomputer I/O interface.

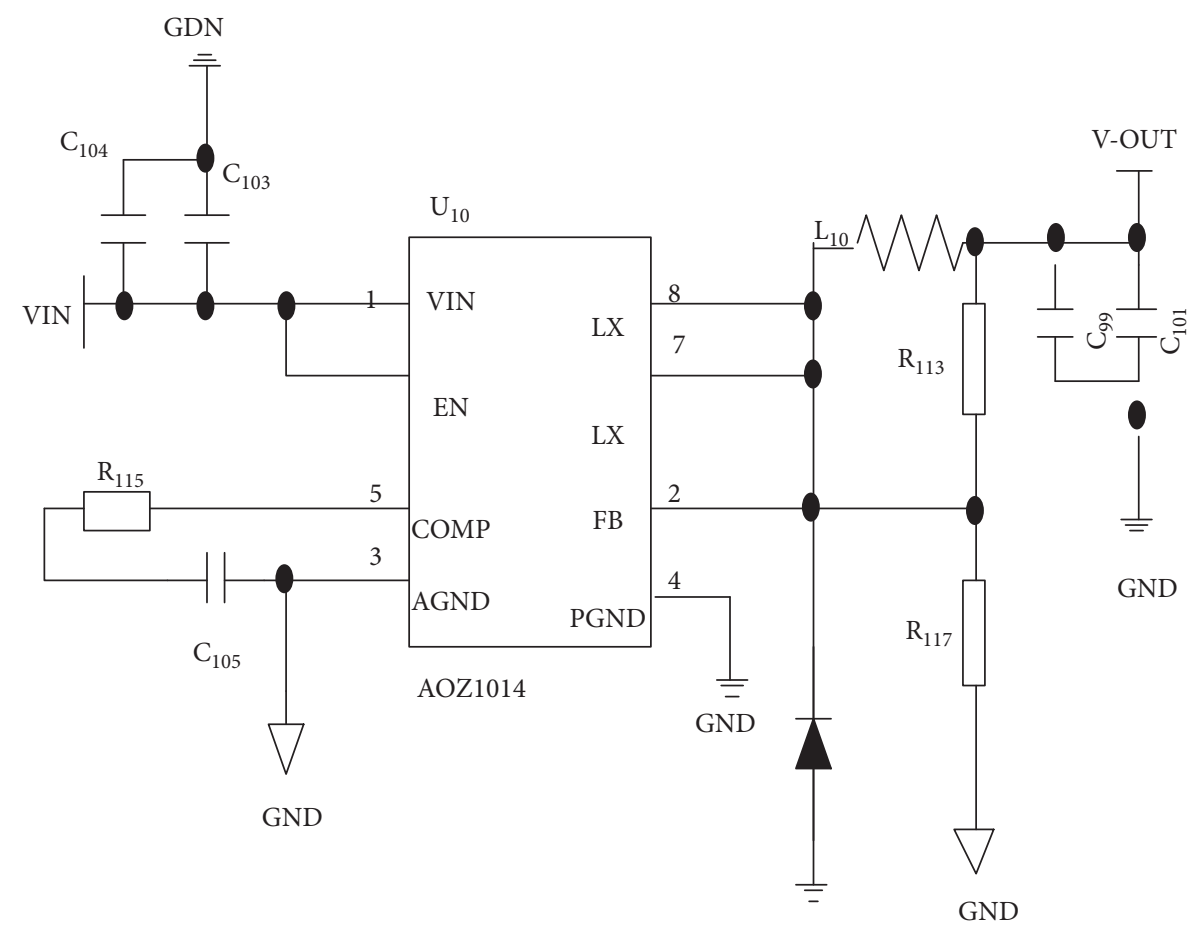

FIGURE 4: Schematic diagram of the interface circuit.

Assuming that $a_{i j}, a_{i k}$ is two categories in the class, the similarity between $\mathrm{B} a_{i j}$ and $a_{i k}$ is analyzed according to the data properties and within-data structural relationship characteristics, whose expression is

$$
\operatorname{Sim}\left(a_{i j}, a_{i k}\right)=\mu \operatorname{Sim}_{A}\left(a_{i j}, a_{i k}\right)+\delta \operatorname{Sim}_{\mathrm{intra}}\left(a_{i j}, a_{i k}\right) \text {. }
$$

If there is a similar relationship between resource data characteristics and between $E_{i}, E_{j}, H=1$; if not, $H=0$.

Based on the determination of the similarity of the above resource data, the data with higher similarity shall be fused; that is, 


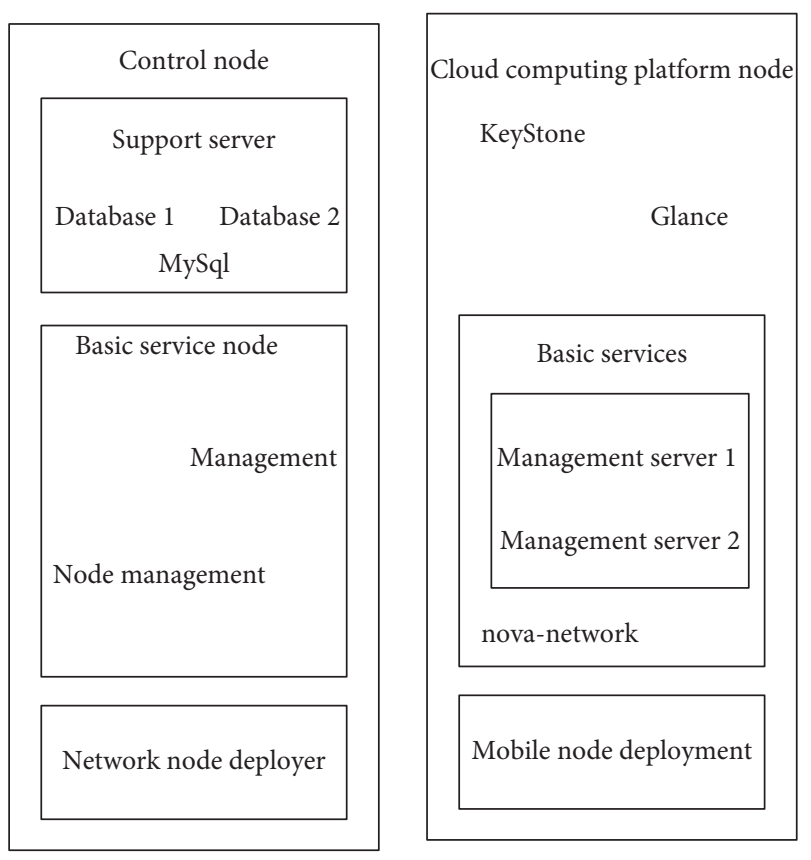

Figure 5: Schematic diagram of node deployment in cloud computing platform.

$$
P\left(E_{i}, E_{j}\right)=\sum_{r=1}^{p}\left|E_{i}^{r}-E_{j}^{r}\right|+\omega \sum_{r=p+1}^{N} \phi\left(E_{i}^{r}, E_{j}^{r}\right) .
$$

In formula (4), $E_{i}^{r}, E_{j i}^{r}$ represents the different attribute properties of different resource data, $N$ represents the total number of fused similar resource data, and $₫$ represents the coefficient of difference between resource data.

Based on the above resource data processing in the cloud platform, the stored data is further processed with the help of a neural network to reduce the redundancy in the data and improve the performance of the cloud computing platform.

A neural network is composed of an input layer, hidden layer, and output layer, with one input layer, one output layer, and multiple hidden layers. All layers except the nodes in the same layer are connected by neuron nodes. There is no coupling between nodes in the same layer, and the neurons in each layer are only sensitive to the input of neurons in the previous layer. The output of neurons in each layer only affects the output of the next layer. The basic structure of data stored in the neural network processing platform is shown in Figure 6.

Enter the cloud platform storage-related data in the neural network input layer, mainly including resource data from the network, network resource buffer data, and relevant cloud platform data uploaded by users. The number of data was processed from specific sources and first obtained by a linear transformation function.

In the resource data processing layer of the cloud platform, the nonlinear transformation function is applied to describe the interaction between input processing units, and the overlapping similar or the same data are deleted; namely,

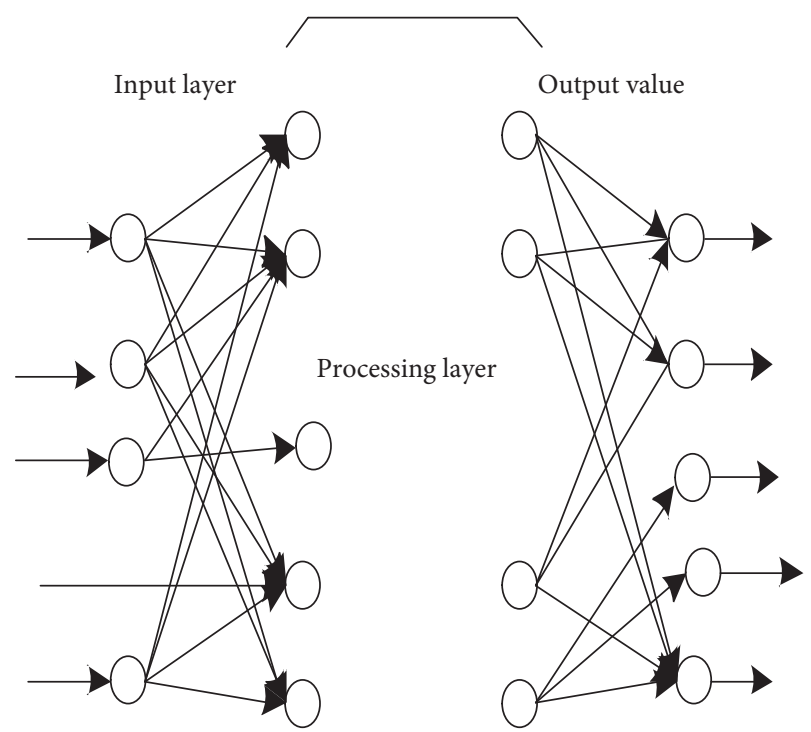

FIGURE 6: Basic data storage structure of neural network processing platform.

$$
W_{i}=\frac{V_{i}}{\sum_{j=1}^{n} V_{i}} .
$$

In formula (5), $W_{i}$ represents the actual value after removing the data and $V_{i}$ represents the number of similar data.

In the neural network structure, the processing layer in the remaining data input structure; namely,

$$
U=(F+K) / 2 \text { or } K=\left(I^{*} K\right)^{1 / 2} \text {. }
$$

In this formula, $L$ is different node data representing the input neural network processing layer.

In the data processing layer, the weight of the input data is obtained, and the weight is

$$
Q_{i}=q_{i}^{\prime}+\Delta q
$$

where $\Delta q$ represents the random perturbation value of the data in the processing layer and the data in the processing layer is processed by the objective function; that is,

$$
\wp(x)=\frac{1}{2 n} \sum_{k=1}^{n}\left[q_{i}(a)-y(k)\right]^{2} .
$$

In this formula, $n$ represents the number of data in the processing layer, $q_{i}(a)$ represents the actual amount of data, and $y(k)$ represents the actual output value of the data.

The implementation process of neural network processing cloud platform data storage is shown in Figure 7.

\section{Experimental Analysis}

A method of experimental psychology that focuses on individual behavior rather than group averages in order to investigate the links between specific experiences and changes in behavior. In this section, we explain the experimental scheme, design of experimental indicators, and analysis of experimental results in detail. 


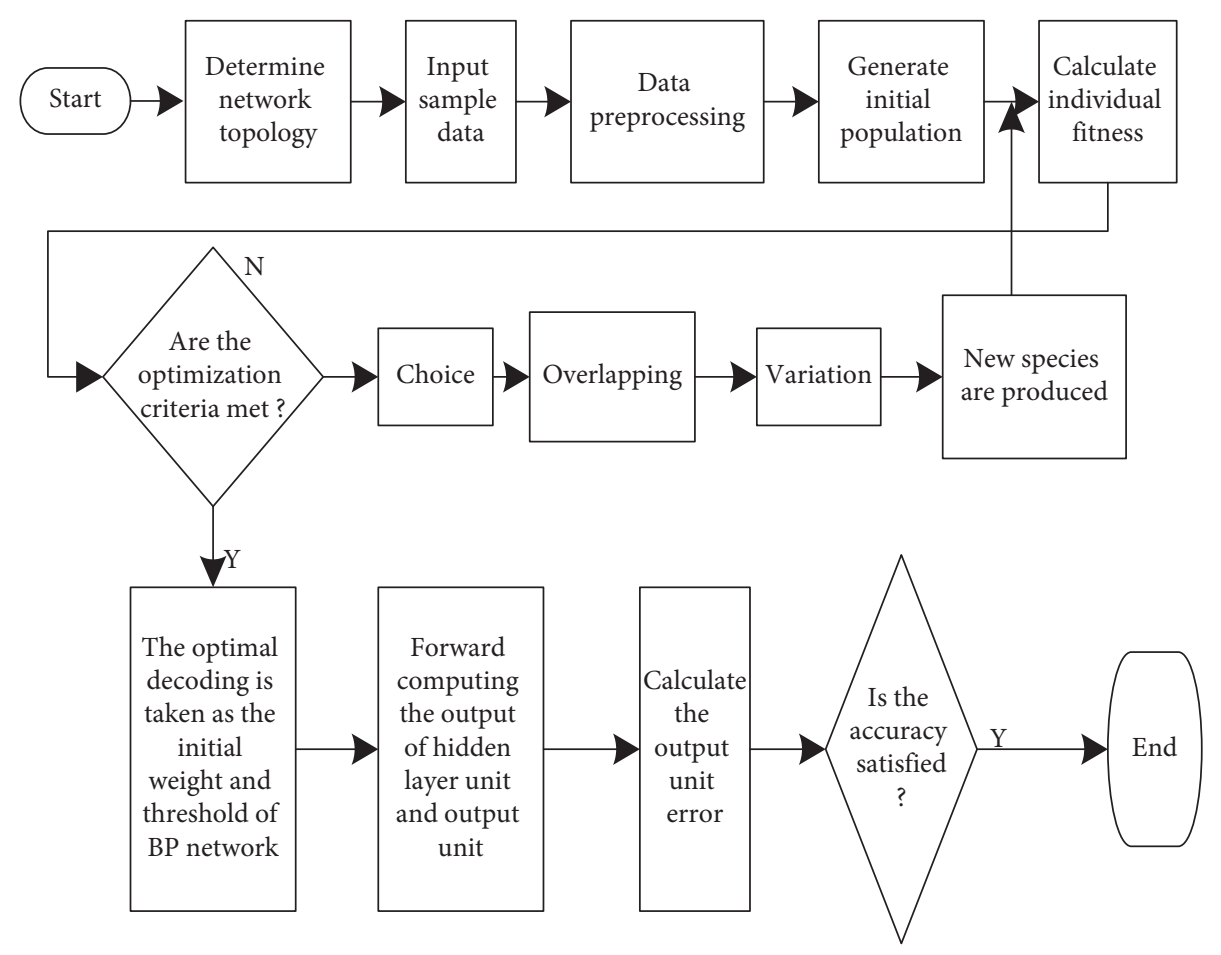

FIGURE 7: Implementation flow of neural network algorithm.

3.1. Experimental Scheme. In order to verify the effectiveness of the proposed method, the performance of the designed platform is tested. In the test, the platform is developed on the basis of basic hardware. The developed platform can run normally, and its memory for storing data is large, which is in line with the research of this experiment. In the experiment, the data resources to be stored are selected from the MySQL database, and $1 \mathrm{~GB}$ of data is selected for storage. There is a certain amount of duplicate data and honor data in the data, which needs to be processed when stored. The interval of experimental data storage is $0.5 \mathrm{~s} /$ time. The operating environment of the experimental development platform is shown in Figure 8.

3.2. Design of Experimental Indicators. In the design of experimental indicators, the amount of data stored on the platform, the small throughput of the platform, and the energy consumption of the platform are taken as the experimental indicators. The experiment is carried out in the form of comparison. In the comparison, this paper takes the platform of this paper, the platform of literature [8], the platform of literature [9], and the platform of literature [10] as the comparison object and compares the experimental results obtained by the four developed platforms on the basis of experimental indicators, so as to highlight the effectiveness of this method.

\subsection{Analysis of Experimental Results}

3.3.1. Comparison of Data Storage on Different Platforms. The storage capacity of platform data is a good measure, which can measure the internal storage and data processing

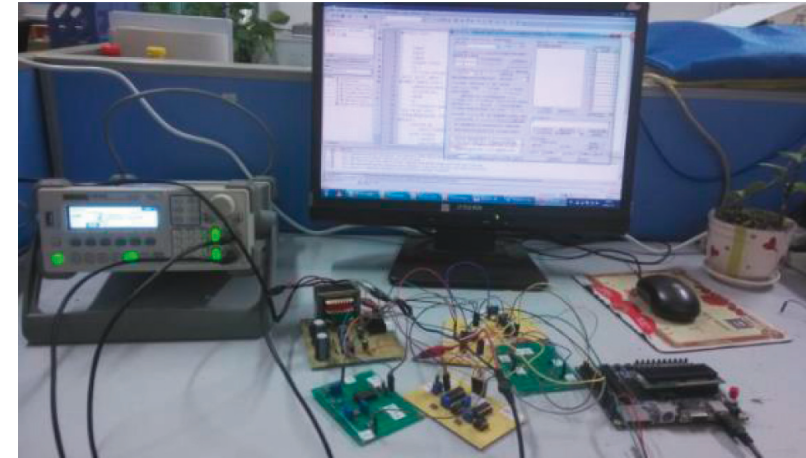

Figure 8: Experimental environment.

capacity of the platform. Therefore, in this experiment, we compared the changes in the storage capacity of this platform, document [8] platform, document [9] platform, and document [10] platform when storing sample data. Taking 5000 pieces of data in the sample data as the research sample, we analyzed the amount of data that can be stored by each platform with the change of iteration times. The results are shown in Figure 9.

By analyzing the experimental results in Figure 9, it can be seen that there are some differences in the change of storage volume when storing sample data using this platform, literature [8] platform, literature [9] platform, and literature [10] platform. When the number of iterations is 50, the sample data stored on the platform of this paper is about 4800 , the sample data stored on the platform of literature [8] is about 3700, the sample data stored on the platform of literature [9] is about 3200, and the sample data stored on the platform of literature [10] is about 2500. When the number 


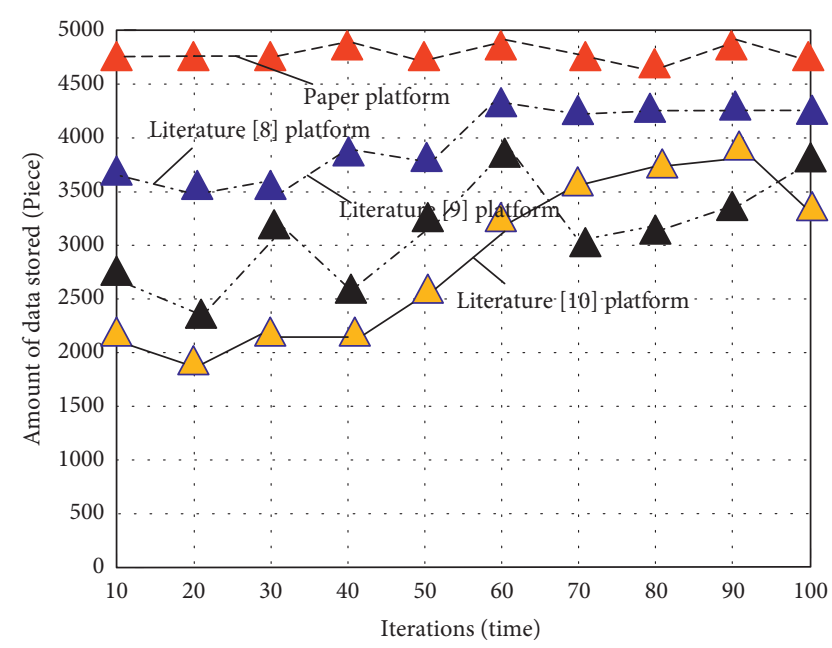

Figure 9: Comparison results of data storage on different platforms.

of iterations is 100 , the sample data stored on the platform of this paper is about 4700 , the sample data stored on the platform of literature [8] is about 4200, the sample data stored on the platform of literature [9] is about 3100, and the sample data stored on the platform of literature [10] is about 3700. Through the comparison of data, it can be seen that, with the continuous change of iteration times, the amount of data stored on the four platforms has changed to some extent. However, from the overall trend, the amount of data stored on the platform in this paper is greater than that on the other three platforms. This is because the platform in this paper considers the problems of system memory and data storage in the development, which improves the effectiveness of the platform in this paper, and the practical operability of the platform is verified.

\subsubsection{Comparative Analysis of Network Throughput on} Different Platforms. In order to verify the performance of the designed platform, the experiment further analyzes the network throughput performance of this platform, reference [8] platform, reference [9] platform, and reference [10] platform. The larger the throughput, the better the performance of this platform. Therefore, the network throughput of the four platforms is experimentally analyzed, and the results are shown in Figure 10.

From the experimental results in Figure 10, it can be seen that there are some differences in the network throughput performance of this platform, literature [8] platform, literature [9] platform, and literature [10] platform. When the number of iterations is 40 , the platform network throughput in this paper is about $2200 \mathrm{Mpbs}$, the platform network throughput in literature [8] is about $510 \mathrm{Mpbs}$, the platform network throughput in literature [9] is about $480 \mathrm{Mpbs}$, and the platform network throughput in literature [10] is about $600 \mathrm{Mpbs}$. When the number of iterations is 100 , the platform network throughput of this paper is about $2500 \mathrm{Mpbs}$, the platform network throughput of literature [8] is about $2000 \mathrm{Mpbs}$, the platform network throughput of

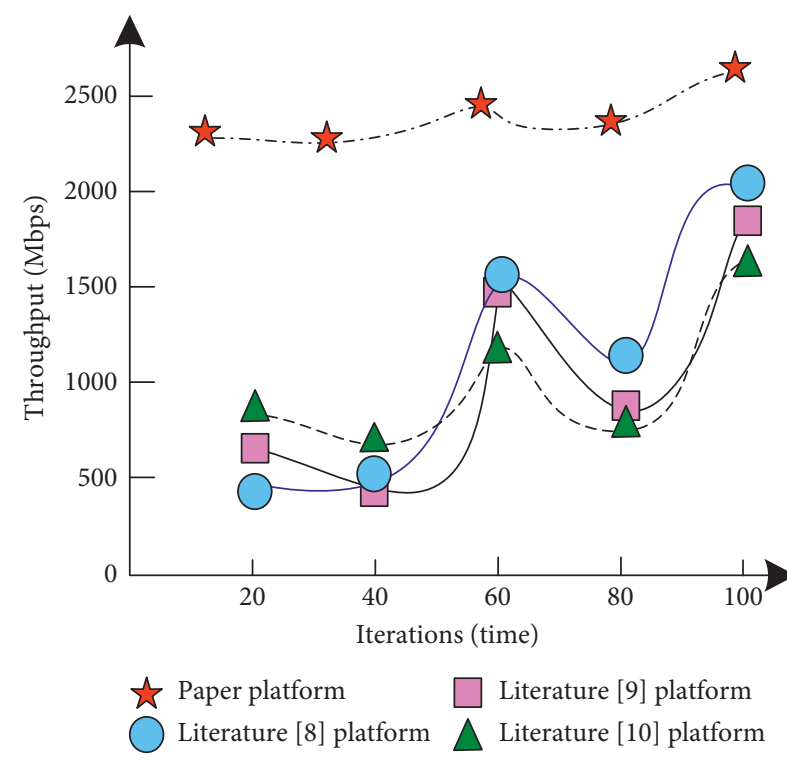

FIGURE 10: Analysis of network throughput comparison results of different platforms.

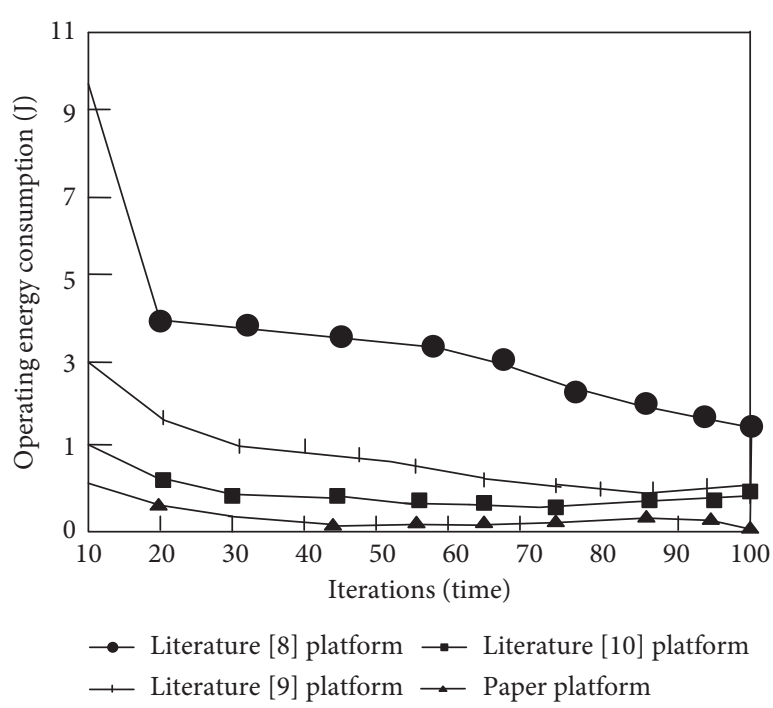

FIgURE 11: Analysis of operation energy consumption of different platforms.

literature [9] is about $1800 \mathrm{Mpbs}$, and the platform network throughput of literature [10] is about $1600 \mathrm{Mpbs}$. In contrast, the performance of this platform is better.

\subsubsection{Energy Consumption Analysis of Different Platforms.} Operation energy consumption is also the key to measuring the performance of the platform. Therefore, the energy consumption of this platform, reference [8] platform, reference [9] platform, and reference [10] platform is experimentally analyzed. The lower the energy consumption, the better the performance. The experimental results are shown in Figure 11.

By analyzing the experimental results in Figure 11, it can be seen that there are some differences in the operating 
energy consumption of the four platforms in the operating energy consumption test by using the platform of this paper, the platform of literature [8], the platform of literature [9], and the platform of literature [10]. Among them, the operation energy consumption of the platform in this paper is lower and always shows a downward trend, while the operation energy consumption of the other three traditional platforms is higher than that of this platform, which verifies the effectiveness of this platform.

\section{Conclusion}

This study develops a cloud computing platform based on neural networks to overcome the challenges of tiny data storage, low platform throughput, and high energy consumption in the development of existing cloud computing platforms. The functions of the cloud computing platform are first identified during platform design. The hardware and software of the cloud computing platform are created based on the function design. The structure of the cloud computing platform, data acquisition module, single-chip microcomputer, node deployment, and other types of functional hardware are designed in the hardware design phase. The neural network is mostly used in software design to reduce redundancy from data stored in a cloud computing platform, design the cloud computing platform's data processing flow, and finish the construction of a cloud computing platform based on a neural network.

The experimental results show the following:

(1) The amount of resource data stored on this platform is large and operable

(2) When using this platform, the network throughput is significantly improved, and the effect is good

(3) The energy consumption of this platform is always lower than that of the traditional three platforms, which verifies the effectiveness of this method

\section{Data Availability}

The data used to support the findings of this study are available from the corresponding author upon request.

\section{Conflicts of Interest}

The authors declare that they have no conflicts of interest.

\section{References}

[1] H. Kai, V. Hannes, B. P. Torben, and L. Wolfgang, "Multischema-version data management: data independence in the twenty-first century.[J]," VLDB J, vol. 27, no. 4, pp. 547-571, 2018.

[2] S. Sandya and Athukoralalage, "Towards school assessment resources sharing UsingCloud computing platform[J]," Polymer, vol. 14, no. 02, pp. 1145-1149, 2019.

[3] L. Chen and Y. Li, "Development of Web information database system under iot architecture[J]," Automation \& Instrumentation, vol. 5, no. 08, pp. 113-116, 2018.
[4] G. Han, Y. Li, and Y. Gao, "Design and development of MySQL database based on embedded Qt[J]," Microcomputer Applications, vol. 36, no. 05, pp. 25-27, 2020.

[5] T. C. Lin, Y. Jun-Zhe, and C. S. Yu, "Development of a transmission network fault location platform based on cloud computing and synchrophasors[J]," IEEE Transactions on Power Delivery, vol. 14, no. 29, pp. 1-6, 2019.

[6] G. Li, X. Zhou, J. Sun et al., "A survey of machine learning based database techniques[J]," Chinese Journal of Computers, vol. 43, no. 11, pp. 2019-2049, 2020.

[7] A. Abdulhameed, "Evaluating distributed IoT databases for edge/cloud platforms using the analytic hierarchy process[J]," Journal of Parallel and Distributed Computing, vol. 124, pp. 41-46, 2018.

[8] X. Pan, S. Xu, X. Cai, Y. Wen, and X. Yuan, "Survey on deep learning based natural language interface to database[J]," Journal of Computer Research and Development, vol. 58, no. 09, pp. 1925-1950, 2021.

[9] Y. Liu, C. Sha, and J. Niu, "analysis of topics on database systems in stack overflow[J]," Computer Science, vol. 48, no. 06, pp. 48-56, 2021.

[10] X. Wang, L. Zhang, and L. Yang, "Artificial intelligence and database technology work together[J]," Journal of Physics: Conference Series, vol. 1955, no. 1, pp. 1932-1945, 2021.

[11] Z. P. Luo, Y. C. Jiang, and Z. J. Hu, "Cloud computing virtual resource enhanced multipoint secure transmission simulation [J]," Computer Simulation, vol. 38, no. 1, pp. 158-161, 2021.

[12] Y. Liu, "Method of developing database driven by golang[J]," Computer and Modernization, vol. 6, no. 01, pp. 113-115, 2018.

[13] D. Pooja and S. Arun, "A new software development paradigm for intelligent information systems[J]," International Journal of Intelligent Information and Database Systems, vol. 13, no. 2-4, pp. 356-375, 2020.

[14] Y. Wu, "Database construction in computer audit under big data environment[J]," Journal of Physics: Conference Series, vol. 1881, no. 3, pp. 139-156, 2021.

[15] H. Liang and J. Han, "Research on decentralized transaction consensus mechanism of cloud computing resources based on block chain[J]," Computer Science, vol. 46, no. S2, pp. 548$552,2019$.

[16] W. Doh, H. Kim, K.-b. Choi, and H. C. Choe, "Introduction to metaphor database construction," Korean Semantics, vol. 61, pp. 55-79, 2018.

[17] S. T. Jeyakumar, T. Mukunthan, and M. Vallipuram, "A blockchain-based database management system[J]," The Knowledge Engineering Review, vol. 35, pp. 1278-1288, 2020.

[18] X. S. Cheng, Z. Yang, and J. Wang, "Association rules mining method of frequency hopping data in cloud computing network intrusion[J]," Computer Simulation, vol. 38, no. 3, pp. 259-263, 2021.

[19] M. Villari, M. Fazio, S. Dustdar, O. Rana, D. N. Jha, and R. Ranjan, "Osmosis: the osmotic computing platform for microelements in the cloud, edge, and internet of things," Computer, vol. 52, no. 8, pp. 14-26, 2019.

[20] Z. Qian, X. Wang, X. Liu, X. Xie, and T. Song, “An approach to dynamically assigning cloud resource considering user demand and benefit of cloud platform," Computing, vol. 102, no. 8 , pp. $1817-1842,2020$. 